\title{
Image Enhancement of Sea Objects based on Extended BEMD
}

\author{
Yang Jinyan $^{1}$, Xu Guanlei ${ }^{2}, \mathrm{Li} \mathrm{Yu}^{1}$ \\ ${ }^{1}$ Military physical education room of Dalian Naval Academy, Dalian, 116018, China \\ ${ }^{2}$ Ocean department of Dalian Naval Academy, Dalian, 116018, China \\ xxxggg1110@163.com,xgl_86@163.com
}

\begin{abstract}
The safety is of much importance in sea transport for ships. This paper proposes a new way to enhance the sea objects' images that have low contrast based on extended BEMD (bidimensional empirical mode decomposition). The zero-mean waves are added and subtracted for image decomposition instead of the zero-mean random Gaussian noises as assisted signals. The new proposed method has the better performance in time-cost and complexity. After the decomposition of image to IMFs by the extended BEMD, the details and luminance of these sea objects' images as well as color are modulated to enhance the degraded sea objects' images. The efficiency of the proposed method is shown by some experiments.

Index Terms - Image enhancement; Extended BEMD (bidimensional empirical mode decomposition); Sea objects' images; Zero-mean waves
\end{abstract}

\section{Introduction}

Enhancing the degraded images is an important problem in vision and has many applications [1,2]. For example, we often analyze the sea objects in fog or other bad weather to determine how many objects and what objects in the sea. In ship training and transport, the ships should see each other or other objects such as hills for the safety. Before analysis, the sea objects' images in sea have to be enhanced because of degradation. Such degraded images often lack visual vividness and offer a poor visibility of the scene contents. This effect may be an annoyance to undermine the quality of needed images. These effects result in a loss of contrast that is characteristic of poor visibility conditions. Many algorithms are available for enhancing image contrast such as [3]-[7]. Of these algorithms, perhaps the best one is the histogram equalization. Histogram equalization and its variants may be applied locally but low spatial frequencies are then lost.

In the paper we present a new method to enhance this type of images given a single image as an input. We achieve this by new image decomposition, extended bi-dimensional empirical mode decomposition (extended BEMD), to restore the details and colors that have been degraded for under water images.

\section{EXTENDED BEMD}

The empirical mode decomposition (EMD) has been developed as a valuable time-frequency-magnitude resolution tool for non-stationary signals and been widely explored [8].
And with the development of EMD, the bi-dimensional empirical mode decomposition (BEMD) come into being and get more and more applications such as [9]-[13]. First, review the classical BEMD.

\section{A. Classical BEMD}

(1) Initialization: $\operatorname{res}(x, y)=f(x, y)$. (Now we let $f(x, y)$ denote the image.)

(2) Identify all the minima and maxima of $\operatorname{res}(x, y)$, respectively.

(3) Compute the lower and upper envelopes, $E_{d w}(x, y)$ and $E_{u p}(x, y)$, through interpolating the minima and maxima using 2D interpolation functions, respectively.

(4) Compute the mean of $E_{d w}(x, y)$ and $E_{u p}(x, y)$ : $M_{e n}(x, y)=\left(E_{d w}(x, y)+E_{u p}(x, y)\right) / 2$

(5) Subtract $M_{e n}(x, y)$ from $\operatorname{res}(x, y)$ : $\operatorname{Mod}(x, y)=\operatorname{res}(x, y)-M_{e n}(x, y)$

(6) If $\operatorname{Mod}(x, y)$ is not an IMF, $\operatorname{res}(x, y)=\operatorname{Mod}(x, y)$, turn to step (1).

(7) Obtain the IMF: $\operatorname{imf}(x, y)=\operatorname{Mod}(x, y)$.

(8) $\operatorname{res}(x, y)=\operatorname{res}(x, y)-\operatorname{imf}(x, y)$.

(9) Repeat the steps (1)-(8) until the residue $\operatorname{res}(x, y)$ satisfies the stopping condition (in this paper the fixed number of IMFs is given in advance).

After the sifting process of steps (1)-(9), one has

$$
f(x, y)=\sum_{l=1}^{L} \operatorname{imf}_{l}(x, y)+\operatorname{res}(x, y) .
$$

\section{B. Extended BEMD}

(1) From the extrema of image $f(x, y)$ and the information obtained in section $\mathrm{C}$ to construct the wave image $f_{a}(x, y)$.

(2) Add the wave image $f_{a}(x, y)$ to the targeted image $f(x, y): f_{a}^{+}(x, y)=f(x, y)+f_{a}(x, y)$.

${ }^{*}$ This work is partially supported by NSFC Grant \#61002052 
(3) Decompose the data $f_{a}^{+}(x, y)$ using the classical BEMD : $f_{a}^{+}(x, y)=i m f_{j}^{+}(x, y)+\operatorname{res}^{+}(x, y)$

(4) Subtract the wave image $f_{a}(x, y)$ from the targeted image $f(x, y): f_{a}^{-}(x, y)=f(x, y)-f_{a}(x, y)$.

(5) Decompose the data $f_{a}^{-}(x, y)$ using the classical BEMD : $f_{a}^{-}(x, y)=\operatorname{imf}_{j}^{-}(x, y)+\operatorname{res}^{-}(x, y)$

(6) The mean:

$$
\operatorname{imf}_{j}(x, y)=\frac{i m f_{j}^{+}(x, y)+i m f_{j}^{-}(x, y)}{2},
$$

$$
\operatorname{res}(x, y)=\frac{\operatorname{res}^{+}(x, y)+\operatorname{res}^{-}(x, y)}{2}
$$

(7) If $r e s(x, y)$ is not a eligible residue, then set $f(x, y)=\operatorname{res}(x, y)$ and turn to step (1), otherwise

$$
f(x, y)=\sum_{j=1}^{L} \operatorname{imf}_{j}(x, y, J)+\operatorname{res}(x, y) .
$$

Similarly with [6], considering the ASBEMD approach and the relation $f_{a}^{+}(x, y)=i m f_{j}^{+}(x, y)+\operatorname{res}^{+}(x, y)$, the equation in step (2) of ASBEMD can also be rewritten as

$$
\begin{aligned}
& f_{a}^{+}(x, y)=i m f_{j}^{+}(x, y)+\operatorname{res}^{+}(x, y) \\
& =\left.\left\{\operatorname{imf}_{j}^{+}(x, y)+\operatorname{res}^{+}(x, y)\right\}\right|_{f(x, y)} \\
& +\left.\left\{\operatorname{imf}_{j}^{+}(x, y)+\operatorname{res}^{+}(x, y)\right\}\right|_{f_{a}(x, y)} .
\end{aligned}
$$

In the same manner, we have

$$
\begin{aligned}
& f_{a}^{-}(x, y)=i m f_{j}^{-}(x, y)+\operatorname{res}^{-}(x, y) \\
& =\left.\left\{i m f_{j}^{-}(x, y)+\operatorname{res}^{-}(x, y)\right\}\right|_{f(x, y)} \\
& +\left.\left\{i m f_{j}^{-}(x, y)+\operatorname{res}^{-}(x, y)\right\}\right|_{f_{a}(x, y)} .
\end{aligned}
$$

Also, we know that

$$
\begin{aligned}
& \left.\left\{\operatorname{imf}_{j}^{-}(x, y)+\operatorname{res}^{-}(x, y)\right\}\right|_{f(x, y)}=\left.\left\{\operatorname{imf}_{j}^{+}(x, y)+\operatorname{res}^{+}(x, y)\right\}\right|_{f(x, y)}, \\
& \left.\left\{\operatorname{imf}_{j}^{+}(x, y)+\operatorname{res}^{+}(x, y)\right\}\right|_{f_{a}(x, y)}=-\left.\left\{i m f_{j}^{-}(x, y)+\operatorname{res}^{-}(x, y)\right\}\right|_{f_{a}(x, y)} .
\end{aligned}
$$

Finally, we find that the IMFs and residue can reconstruct the original image $f(x, y)$ perfectly using extended BEMD, and there is no difference between the original image $f(x, y)$ and the reconstructed image.

Moreover, we compare the time cost with the classical ASBEMD defined in [6], and table 1 is the result. Now we find that the Extended BEMD save more time, and the timecost of the proposed method is fixed, which is unlike the changing time-cost of the method in [6] with the variance changing of noises. Note that the comparison setting is the same as that of [6].
TABLE I The time cost comparison for ASBEMD and extended BEMD.

\begin{tabular}{|c|c|c|c|c|c|c|c|c|c|c|}
\hline \multicolumn{2}{|c|}{} & \multicolumn{10}{|c|}{$\sigma_{x, y}$} \\
\cline { 2 - 11 } \multicolumn{2}{|c|}{} & 0 & 1 & 3 & 5 & 10 & 15 & 20 & 30 & 50 \\
\hline \multirow{2}{*}{$\begin{array}{c}\text { time } \\
\text { (s) }\end{array}$} & ASBEMD & 7 & 16 & 45 & 49 & 112 & 234 & 355 & 571 & 938 \\
\cline { 2 - 9 } & $\begin{array}{c}\text { Extended } \\
\text { BEMD }\end{array}$ & \multicolumn{10}{|c|}{7} \\
\hline
\end{tabular}

\section{Construction of Wave Image}

In EMD method, Since IMF is based on the local extrema, it always extracts the highest-frequency component. Therefore, if we add a higher-frequency component $f_{a}(x, y)$ to the image $f(x, y)$ to be decomposed, the first IMF $\operatorname{imf}_{1}(x, y)$ will contain the added higher-frequency component at least, and sometimes for further the first IMF will contain the added higher-frequency component and part of the image with the closer frequencies to that of the added higherfrequency component, i.e., $\operatorname{imf} f_{1}(x, y)=f_{a}(x, y)+\left.P(x, y)\right|_{f(x, y)}$ (where $\left.P(x, y)\right|_{f(x, y)}$ denotes the part coming from $f(x, y)$ ). If we remove the added higher-frequency component $f_{a}(x, y)$ from the first IMF $\operatorname{imf}_{1}(x, y)$, then the remainder will be the part $\left(\left.P(x, y)\right|_{f(x, y)}\right)$ of the image with higher frequencies closer to that of the added higher-frequency component. On the other hand, through manipulating the frequency of $f_{a}(x, y)$, we can obtain different part $\left.P(x, y)\right|_{f(x, y)}$ from image $f(x, y)$. For example, if we make the assisted signal $f_{a}(x, y)$ have more overlapped frequencies with image $f(x, y)$, then $\left.P(x, y)\right|_{f(x, y)}$ will contain more parts coming from $f(x, y)$, and vice verse. Through this manner, one can use well manipulated assisted signals to obtain the required components with different bandwidth from image $f(x, y)$. However, in order to remove the assisted signal wholly from the IMF, the whole assisted signal must be included in only one IMF, and thus empirically the local frequencies of the assisted signal $f_{a}(x, y)$ are not less than the local max frequencies at least, and the decomposition efficiency will be better. Otherwise, the assisted signals cannot be removed wholly from the according IMFs. Therefore, we can construct the wave image with the similar method as that in [5].

\section{Image Enhancement for Sea Objects}

\section{A. The enhancement Algorithm}

A new enhancement method based on extended BEMD is proposed for image enhancement in this paper. This enhancement method is based on the decomposition of extended BEMD described above. As one new and developing decomposition method, the most important thing of Extended BEMD involves finding out its valuable applications in image processing. In addition, instead of complex nonlinear 
transform as other enhancement methods in spatial domain, here only a simple linear transform is adopted to enhance the images. After decomposition, we get the result defined in equation (1). The enhanced image $f_{e n}(x, y)$ is obtained by

$$
f e n(x, y)=\sum_{l=1}^{L} \lambda_{l} \cdot \operatorname{imf}_{l}(x, y)+\xi \cdot \operatorname{res}(x, y),
$$

where $0.2 \leq \xi \leq 5, \lambda_{l} \geq 1(l=1,2,3, \cdots, L)$. The pixel value lies in 0 and 1 here. The decomposition of extended BEMD is for $L=5$ and sifting number equal to 5 . $\lambda_{l}$ is used to boost the details (or edges) of different levels. $\lambda_{l}$ determines the boosting degree for the details of level $l$. $\xi$ is used to manipulate the whole luminance of the image. Different images should be taken into account separately. One simple way is to try these parameters first, and then obtain the optimal selections. Generally, $\lambda_{l}$ plays the role of local details manipulation and $\xi$ plays the role of whole global luminance manipulation. For color images, we generally first turn the RGB to HSV, then apply extended BEMD on the V space. The color can be manipulated by $\mathrm{S}$ space. Also, we can take very space $(R, G, B)$ as a single luminance space. In practice, we need to apply different method according to the requirements.

\section{B. Experiments in Image Enhancement}

In fig.1, there are some examples of enhancement results for different parameters compared with other methods to enhance images. Clearly, the details manipulation is mainly performed by $\lambda_{l}$ and the whole luminance manipulation is mainly exhibited by $\xi$, respectively. In color images, the three channels, R, G and B, are operated separately in the same manner.

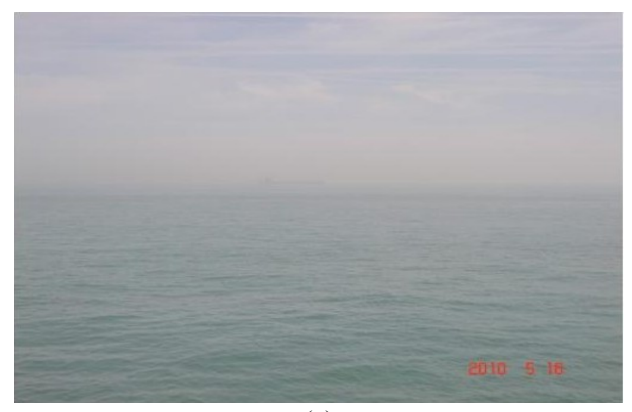

(a)

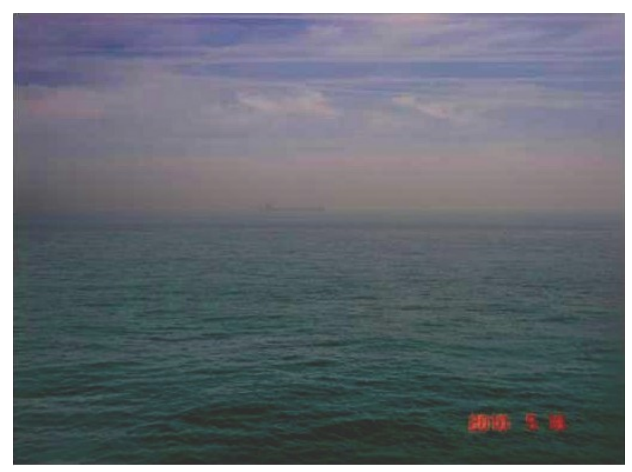

(b)

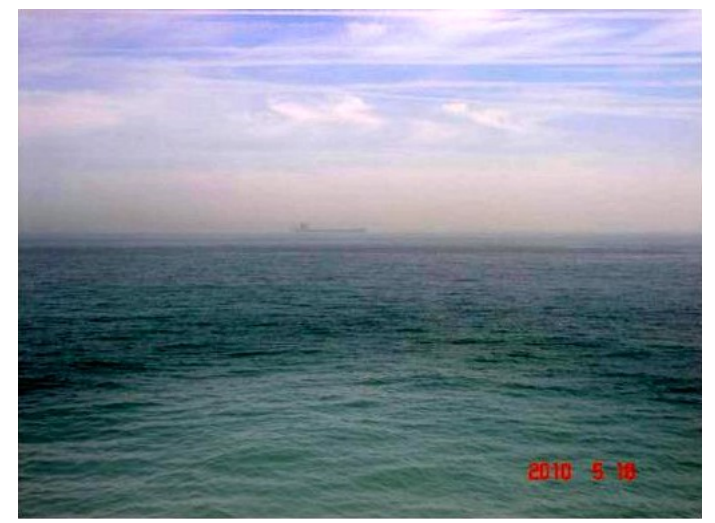

(c)

Fig.1 The comparisons.

(a)The original $(500 \times 600$ pixels); (b)The result of [6]; (d) Our result.

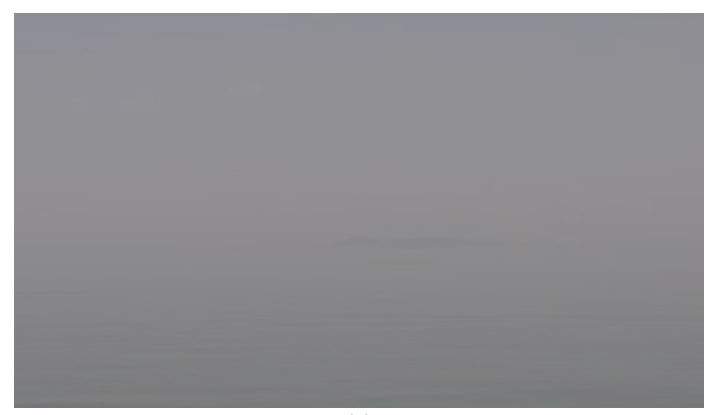

(a)

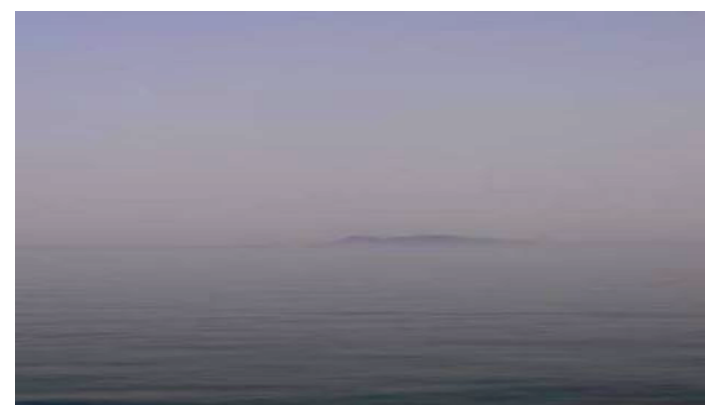

(b)

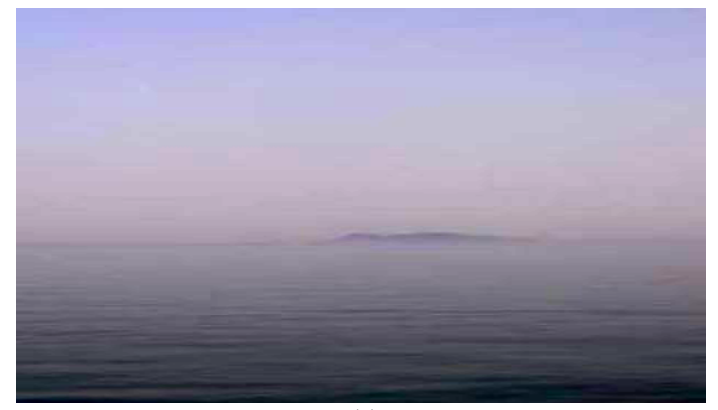

(c)

Fig. 2 The comparisons.

(a)The original $(600 \times 500$ pixels); (b)The result of [6]; (d) Our result.

Clearly, compared with other methods, our enhancement results have better detail boosting and less artifacts (also see fig.2), and the sea objects can be seen more clearly. 


\section{Conclusions}

A new enhancement method based on extended BEMD is proposed for image enhancement of sea objects in this paper. This enhancement method is based on the proposed decomposition of our extended BEMD described above. Rather than using the Gaussian noises as assisted signals, the zero-mean waves are adopted for image decomposition. The time-cost is greatly reduced (see table 1 of the reduction of time cost) with the similar decomposition performance. Then the assisted waves are used to decompose the image to IMFs by BEMD. One can manipulate the details and luminance as well as color to enhance the degraded images. The efficiency of the proposed method is shown via experiments of sea objects. This method will be very useful for the safe transport in sea.

\section{Acknowledgment}

We will thank Professor Zhang Hengduo for his help of this paper in potential application in military physical training.

\section{References}

[1] RC Gonzalez. Digital image processing (second edition).Beijing: Publishing House of Electronics Industry, 2002.

[2] Ali Erol, George Bebis, Mircea Nicolescu ,Richard D. Boyle, Xander Twombly,Vision-based hand pose estimation: A review, Computer Vision and Image Understanding, 108 (2007) 52-73.

[3] Z. Rahman, D. J. Jobson, and G. A. Woodell, "Multi-scale retinex for color image enhancement," IEEE Int. Conf. Image Processing, vol.3, pp.1003-1006, 1996.

[4] C. Munteanu and A. Rosa, "Color image enhancement using evolutionary principles and the retinex theory of color constancy," Proc. 2001 IEEE Signal Processing Society Workshop on Neural Networks for Signal Processing XI, pp. 393-402, 2001.
[5] QIAN Zhibo, ZHOU Lijia, XU Guanlei, Bandlimited BEMD and Application in Remote Sensing Image Fusion, The International Conference on Remote Sensing, Environment and Transportation Engineering, 2011, 2979 - 2982.

[6] G.L. Xu, X.T. Wang, X.G. Xu, "Improved Bi-Dimensional Empirical Mode Decomposition based on 2D Assisted Signals: Analysis and Application, 'IET Image Processing, 2011,5(3):205-221.

[7] J. Starck, F. Murtagh, E.J. Candès, and D. L. Donoho, "Gray and color image contrast enhancement by the Curvelet transform," IEEE Trans. Image Processing, vol. 12, No. 6, pp.706-717, 2003.

[8] N. E. Huang, Z. Shen, S. R. Long, M. C.Wu, et al., "The empirical mode decompositionand the Hilbert spectrum for nonlinear and nonstationary time seriesanalysis," Proc. R. Soc. Lond. A, vol. 454, pp. 903-995, 1998.

[9] J.C. Nunes, O. Niang, Y. Bouaoune, E. Delechelle, et al., "Texture analysis based on the bidimensional empirical mode decomposition with gray-level co-occurrence models," IEEE, Machine Vision and Application, 2, pp. $633-635,2003$.

[10] Z.H. Yang, D. X. Qi, L.H. Yang, "Signal period analysis based on Hilbert-Huang transform and its application to texture analysis,"IEEE, Proceedings of the Third International Conference on Image and Graphics, pp.430 - 433, 2004.

[11] G.L. Xu, X.T. Wang, X.G. Xu, "Neighborhood limited empirical mode decomposition and application in image processing, " Fourth International Conference on Image and Graphics, 2007, Chengdu of China, pp.149-154.

[12] Xu G.L., Wang X.T., Xu X.G., "Improved bi-dimensional EMD and Hilbert spectrum for the analysis of textures, "Pattern Recognition, 42(5), pp. 718-734,2009.

[13] Xu Guanlei, Wang Xiaotong, Xu Xiaogang. On Analysis of Bidimensional Component Decomposition via BEMD[J]. Pattern Recognition,2012,45(4):1617-1626. 